\title{
Expression of Aquaporins in the Rat Choroid Plexus
}

\author{
Zahra Nazari ${ }^{1}$; Mohammad Nabiuni ${ }^{1, *}$; Zahra Safaei Nejad ${ }^{1}$; Bahram Delfan $^{2}$; Saeed Irian ${ }^{1}$ \\ ${ }_{2}^{1}$ Department of Cell and Molecular Biology, Faculty of Biological Sciences, Kharazmi University, Tehran, IR Iran \\ ${ }^{2}$ Razi Herbal Medicines Research Center, Lorestan University of Medical Sciences, Khoramabad, IR Iran \\ ${ }^{*}$ Corresponding author: Mohammad Nabiuni, Department of Cell and Molecular Biology, Faculty of Biological Sciences, Kharazmi University, Tehran, IR Iran. Tel: +98-9126609337, \\ Fax: +98-2614510005, E-mail: nabiuni@khu.ac.ir
}

Received: January 5, 2014; Revised: January 11, 2014; Accepted: May 5, 2014

\begin{abstract}
Background:Aquaporins (AQP) are water channels that play a key role in water transport in many epithelia. Recent studies have shown that AQP1, located in the apical membrane of choroid plexus cells, has an important role in cerebrospinal fluid (CSF) production. However, the mechanism of water transport through the basolateral membrane of choroid plexus (CP)cells is yet to be determined.

Objectives: In this study, the expression and localization of AQP1, AQP4 and AQP5 in the rat lateral ventricle CP were investigated by immunocytochemistry, western analysis and flowcytometry.

Materials and Methods: Choroidal epithelial cells of the lateral ventricle in Wistar rats were isolated and grown in Dulbecco's Modified Eagle's medium (DMEM) supplement, which was refreshed every two days. After five days, the expression patterns of AQP1, AQP4 and AQP5 were determined by immunoblotting, immuncytochemistry and flowcytometry.

Results: The immunocytochemistry data revealed the expression of AQP1 and AQP4 in the membrane and the cytoplasmof AP cells, respectively. Through western analysis, the AQP1 antibody detected two bands of approximately 27 and $32 \mathrm{kDa}$. A single peptide of $29 \mathrm{kDa}$ was recognized by the AQP4 antibody. Quantitative flowcytometry revealed CP cells exhibiting a high level of AQP1 and AQP4 proteins (95.39\% and 92.21\%, respectively). According to immunocytochemistry, AQP5 is weakly expressed in the cytoplasm of CP cells. Anti-AQP5 antibody recognized a pale band of approximately $28 \mathrm{kDa}$ by western analysis.

Conclusions: These observations suggested that AQP4 may have an important role in CSF secretion; however, expression of AQP4 or AQP5 was not detected in the cell membrane. Thus, how water crosses the basolateral membrane of CP cells remains to be determined.
\end{abstract}

Keywords:Aquaporins; Cerebrospinal Fluid; Choroid Plexus Cells; Lateral Ventricle; Wistar Rats

\section{Background}

Aquaporins (AQPs), a family of water channel proteins, play a major role in water transport in plasma membrane of many human tissues and cell types (1). Some members of the aquaporin family such as AQP1, AQP4, AQP9 and $A Q P 11$ have been identified in the central nervous system (2). AQP4 and AQP1 play vital roles in the dynamic regulation of brain water homeostasis and the regulation of cerebrospinal fluid (CSF) production (3). AQP1 is abundant on the apical surface of choroid plexus (CP) epithelium, a branched structure made up of a single layer of epithelial cells and fenestrated blood capillaries; CSF is mainly produced by this structure (4). The process of CSF secretion involves regulated transport of $\mathrm{Na}^{+}, \mathrm{Cl}$-and $\mathrm{HCO}$ - from the blood to the ventricles, creating an osmotic gradient that drives the secretion of water through AQPs. Less is known, however, about the route by which water is transported to the CSF (2). Recent data suggests that water transport across the CP epithelium is transcellular (5), and AQP1 plays a major role in mediating water transport from the cell to the CSF, across the apical membrane during CSF se- cretion $(6,7)$. However, the route by which water crosses the basolateral membrane of CP cells remains unknown. AQP4 is the predominant water channel in the brain. This protein is expressed in the basolateral membrane of many epithelia including the kidney collecting duct, colon, trachea, and the basolateral membrane of the ependymal cells lining the brain ventricles $(8,9)$. Thus, AQP4 is a likely candidate for the water transport route in the basolateral membrane of the CP epithelium. However, there are conflicting data on AQP4 expression in the choroid plexus. In several studies, western analysis, immunocytochemical survey and in situ hybridization have demonstrated the expression of AQP4 in the epithelial cells of rat's lateral ventricle choroid plexus $(5,10)$. In contrast, some recent immunofluorescence, RT-PCR and in situ hybridization studies failed to detect AQP4 in the rat lateral ventricle $\mathrm{CP}$ (11-14).

\section{Objectives}

Thus, as an initial step, the present study examined AQP4 expression in lateral ventricles CP. To understand the 
mechanism of CSF secretion, the localization and quantity of AQPs in CP cells has to be determined.

Hence, the second aim of the present study was to investigate the distribution and expression of three AQPs (AQP1, AQP4 and AQP5) in the rat lateral ventricles CP through western analysis, flowcytometry and immunocytochemistry for a better comparison.

\section{Materials and Methods}

\subsection{Materials}

Chemicals were obtained from the following sources: DMEM supplement, fetal bovine serum (FBS) and epithelial growth factor (EGF) from Gibco-invitrogen; cytosine arabinoside, phenyl methyl sulfonyl fluoride (PMSF), protease inhibitor cocktail and poly-L-lysin from Sigma; AQP1, AQP4 and AQP5 primary antibodies, and anti-rabbit IgG secondary antibody from Abcam; NP40 lysis buffer from Invitrogen; biotinylated goat anti-rabbit secondary antibody, Vectastain Avidin Biotin complex- Alkaline Phosphatase (ABC-AP) Reagent kit and Vector Blue-AP Substrate kit from Vector Laboratories; Protein ladder from Fermentase. Other chemicals were obtained from standard sources and were of the highest quality available.

\subsection{Primary Cell Culture}

Primary cultures of epithelial cells from rat CPs were prepared using the method described by Zheng et al. (15) with some modifications. In brief, four to six week-old Wistar rats (either sex) were killed and their brains were exposed.CPs from lateral ventricles were rapidly dissected under a stereomicroscope. Having washed the tissues with Hank's balanced salt solution (HBSS), they were chopped with fine ophthalmologic scissors to fragments of about 1 $\mathrm{mm}$. The minced tissue preparation was mixed with $1 \mathrm{~mL}$ of $0.25 \%$ trypsin and incubated at $37^{\circ} \mathrm{C}$ for 10 minutes. The digestion was stopped when $5 \mathrm{ml}$ of phosphate-buffered saline (PBS) solution was added to the digestion mixture. Following centrifugation (1500 rpm for $5 \mathrm{~min}$ ), the pellet of epithelial clumps was resuspended in PBS and mechanically dissociated by passages through an eighteen-gauge needle. Following re-centrifugation, cells were resuspended in $4 \mathrm{~mL}$ of DMEM supplemented with $10 \%$ FBS, 100 units penicillin per $\mathrm{mL}, 100 \mathrm{pg}$ streptomycin per $\mathrm{mL}, 20 \mu \mathrm{l}$ cytosine arabinoside and $10 \mathrm{ng} / \mathrm{mL}$ EGF. Cells were maintained at $37^{\circ} \mathrm{C}, 5 \% \mathrm{CO}_{2}$ on Poly-L-lysine-coated culture wells, and the medium was changed every 48 hours.

\subsection{Immunocytochemistry}

For immunocytochemical examination of AQP1, AQP4 and AQP5 expression, CP cell cultures were washed with PBS and fixed in 4\% paraformaldehyde (PFA) (30 minutes) followed by three washes with PBS. The wells were rinsed with $0.05 \%$ Triton X-100 and blocked with $1 \%$ bovine serum albumin (BSA) in PBS (45 minutes) prior to incubation with a 1:100 dilution of the primary antibody. Following incubation with the primary antibody for 60 minutes at $37^{\circ} \mathrm{C}$, the cultures were incubated in the dark with a flourescence labeled goat anti-rabbit IgG antibody. for 45 minutes at $37^{\circ} \mathrm{C}$. Finally, the cells were washed in PBS solution and mounted for microscopic visualization.

\subsection{Sodium Dodecyl Sulfate Polyacrylamide Gel Electrophoresis and Western Blot Analysis}

Western analysis was performed to examine AQP1, AQP4 and AQP5 expression in the rat CP epithelium. The CP tissues from lateral ventricles were incubated in $500 \mu \mathrm{L}$ of NP40 lysis buffer (supplemented with 1 mM PMSF and 500 $\mu \mathrm{L}$ of protease inhibitor cocktail 10x) for 10 minutes and centrifuged at 10,000 rpm. The content was measured by Bradford assay, and aliquots were stored at $-70^{\circ} \mathrm{C}$. A protein sample of $35 \mu \mathrm{g}$ was solubilized (1:1) in Laemmli sample buffer [0.32 M Tris ( $\mathrm{pH} 6.8$ ), 5\% (w/v) sodium dodecyl sulfate, $25 \%(\mathrm{v} / \mathrm{v})$ glycerol, $1 \%(\mathrm{w} / \mathrm{v})$ bromphenol blue, $5 \%(\mathrm{v} / \mathrm{v})$ $\beta$-mercaptoethanol] and incubated at $95^{\circ} \mathrm{C}$ for 4 minutes. Protein samples were run on $10 \%$ polyacrylamide mini gels. Proteins were then transferred to nitrocellulose membranes during a three-hour period at $300 \mathrm{~mA}$ and 50 $\mathrm{V}$ using a Bio-Rad trans-blot system. Nitrocellulose sheets were washed withTris-buffered saline-Tween (TBST). After blocking with TBST containing 3\% BSA for 1 hour at room temperature, the membranes were separately incubated with anti-AQP1, anti-AQP4 and anti-AQP5 primary antibodies (1:1000 in Tween-PBS) overnight at $4^{\circ} \mathrm{C}$. After washing, they were incubated with the biotinylated goat anti-rabbit secondary antibody for 1 hour. These membranes were washed again with TBST, prior to incubation in $10 \mathrm{~mL}$ of Vectastain ABC-AmP Reagent for 10 minutes. Following a washing step, the membranes were incubated in the substrate solution, and subsequent to the reaction, the membranes were air-dried.

\subsection{Flowcytometry}

In addition to the qualitative analysis (i.e. western blotting and immunocytochemistry), the expression of AQP1, AQP4 in CP cells was also studied using a quantitative method of flowcytometry. The AQP5 primary antibody (Abcam, ab92320) was not suitable for flowcytometry. Briefly, cells were trypsinized and washed with PBS. Following centrifugation (2000 rpm/5 minutes), the supernatant was aspirated, and $500 \mu \mathrm{L}$ of $0.01 \%$ paraformaldehyde was added to each tube and left for 10 minutes. The cells were washed and resuspended in $0.04 \%$ Triton X-100, and the samples were separately incubated with $100 \mu$ Lof AQP1 or AQP4 primary antibodies (diluated 1:100 in blocking buffer BSA/ PBS 3\%) overnight at $4^{\circ} \mathrm{C}$. Cells were then washed with PBS and centrifuged at $2000 \mathrm{rpm}$ for 5 minutes. These cells were later incubated in the dark with $100 \mu \mathrm{L}$ of fluoresceinlabeled goat anti-rabbit secondary antibody (diluted 1:100 in blocking buffer BSA/PBS 3\%) for 45 minutes at $37^{\circ} \mathrm{C}$. After washing with PBS and centrifugation at $2000 \mathrm{rpm}$ for 
Nazari Z et al.

5 minutes, $500 \mu \mathrm{L}$ of PBS was added to each tube and the samples were immediately subjected to measurement by a Becton Dickinson Fluorescence- activated cell Scan analyzer.

\section{Results}

\subsection{Cell Morphology}

The light microscope micrograph in Figure 1 shows the confluent monolayer of rat CP epithelial cells after five days of culture in 10\% FBS/DMEM. Formation of a polarized monolayer with functional cell attachment occurred by 48 hours. As observed in Figure 1, cells grew as densely packed, polygonal shapes, and produced a monolayer displaying a typical cobblestone appearance.

\subsection{Immunocytochemical Staining}

At first, the expression of AQPs was evaluated in the lateral ventricles $C P$ by Immunocytochemistry. According to the results of this qualitative method, AQP1 is only expressed in the cell membranes (Figure $2 \mathrm{~A}$ ). Immunocytochemistry was also employed to examine the localization of AQP4 and AQP5 within the CP cells. The Immunocytochemical signal with the AQP4 antibody was diffusively distributed throughout the cytoplasm, and there was no evidence for AQP4 expression in the cell membrane (Figure $2 \mathrm{~B}$ ). In addition, AQP5 was weakly expressed in the cytoplasm of the $\mathrm{CP}$ cells (Figure $2 \mathrm{C}$ ).

\subsection{Western Blot Analysis}

Western blot analysis of protein samples prepared from the lateral ventricle choroid plexus tissues, separately probed with the AQP1, AQP4 and AQP5 antibodies, is shown in Figure 3. The AQP1 antibody detected two bands of approximately 27 and $32 \mathrm{kDa}$. These molecular masses related to unglycosylated and glycosylated forms of the AQP1 protein (16). A single peptide of approximately $29 \mathrm{kDa}$ was identified by the AQP4 antibody. Furthermore, AQP5 was detected by western analysis of the $\mathrm{CP}$ tissue. The antibody against AQP5 recognized a pale and slim band of approximately $28 \mathrm{kDa}$.

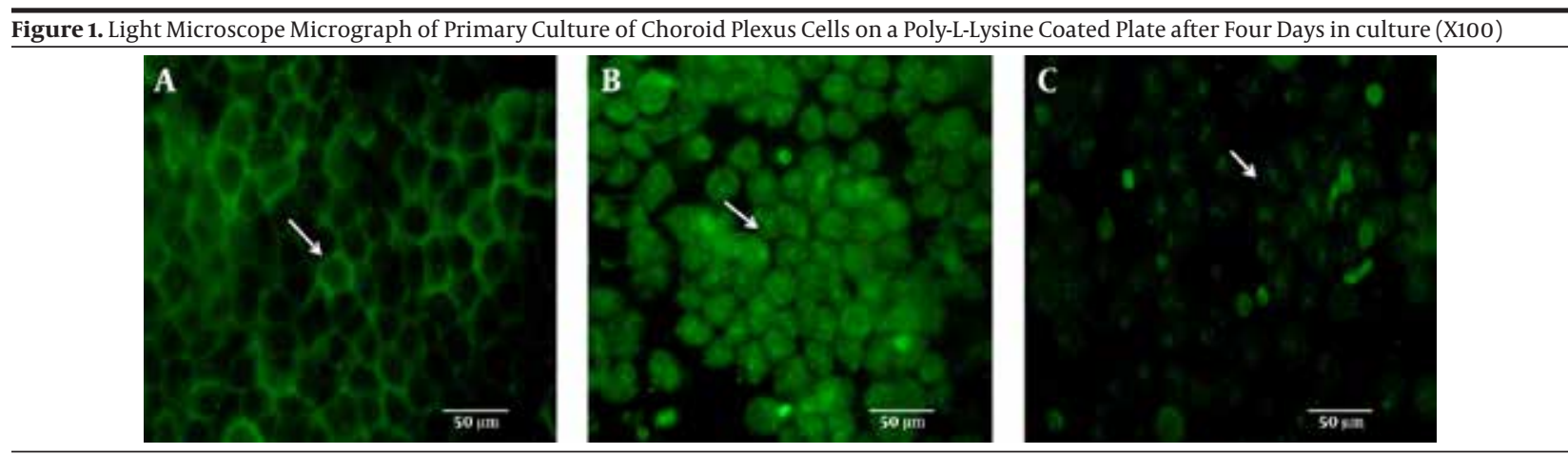

Note the cobblestone appearance of confluent cultured epithelia.

Figure 2. Immunolocalization of Aquaporin1, Aquaporin4 and Aquaporin5Expression in the Rat Lateral Ventricle Choroid Plexus

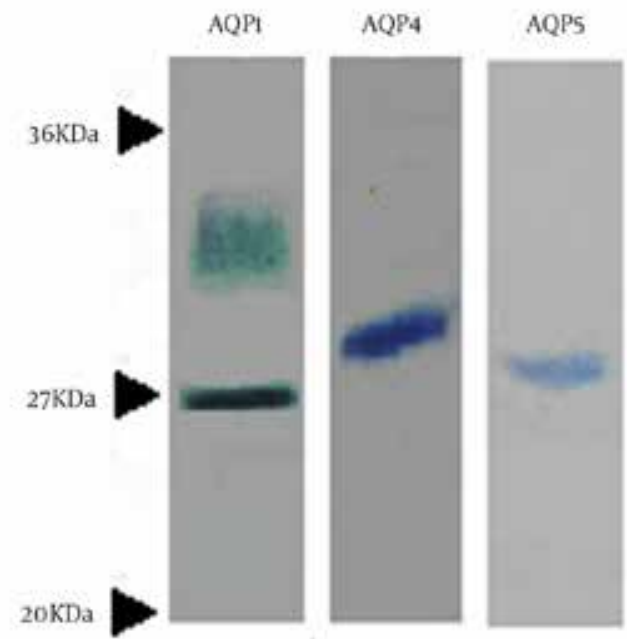

The cells were separately immune labeled with primary antibodies followed by fluorescein isothiocyanate (FITC) conjugated secondary antibody and visualized by fluorescence microscopy at 400x magnification. Arrows indicate the cell membrane. A: the immunoreactivity of APQ1 with the arrow showing that AQP1 is selectively localized in the membrane of choroid plexus cells. B: immunoreactivity of CP cells with an antibody against AQP4, with the arrow showing the unstained cell membrane; an indication that AQP4 expression only occurs in the cytoplasm of the cells. C: expression of AQP5 in CP cells; this protein is weakly expressed in the cytoplasm of CP cells. Arrow points to the lack of expression of AQP5 in the cell membrane. 

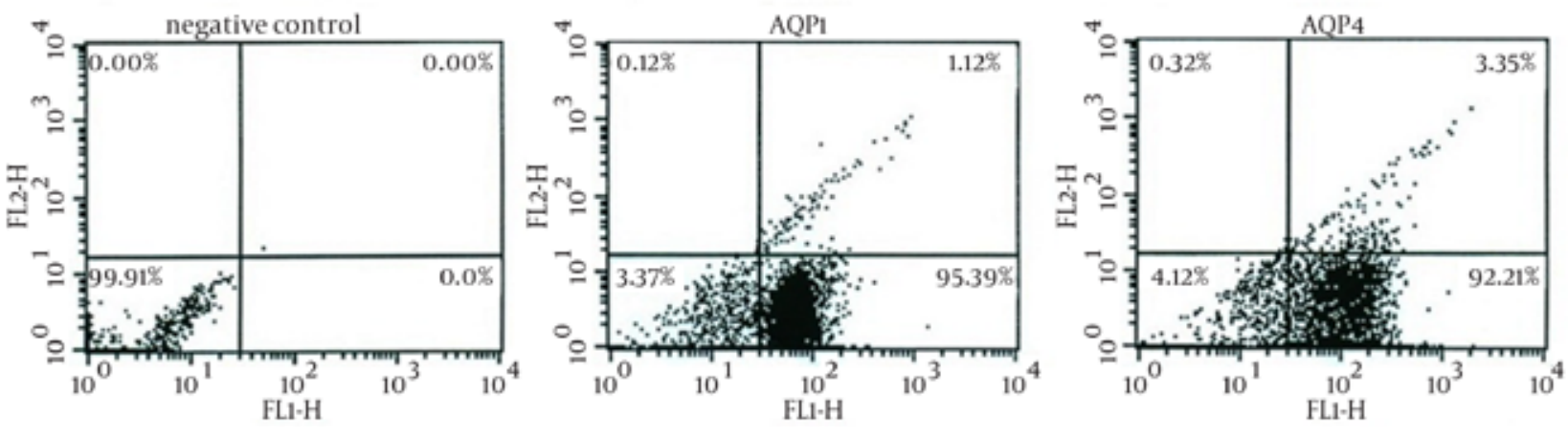

The AQP1 antibody detected two bands of approximately 27 and $32 \mathrm{kDa}$ (Lane 1). A peptide of approximately $29 \mathrm{kDa}$ was identified by the AQP4 antibody (Lane 2). The antibody against AQP5 recognized a slim band of approximately28 kDa (Lane 3).

\subsection{Flow Cytometry}

Flowcytometry revealed CP cells exhibiting a high level of AQP1 and AQP4 proteins (95.39\% and 92.21\%, respectively), compared with the negative control (Figure 4). The AQP5 primary antibody (Abcam, ab92320) was not suitable for flowcytometry. The flowcytometry results correlated with the immunocytochemistry images and Immunoblot bands, which revealed a high level of AQP1 and AQP4 proteins in CP cells.

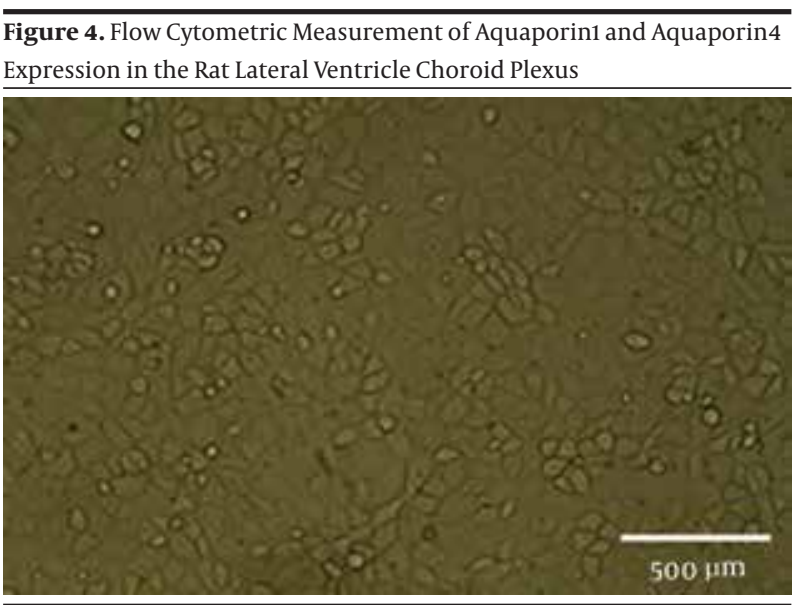

Cells were stained with the primary antibody followed by FITC conjugated secondary antibody and analyzed with a flow cytometer. Results are expressed as relative fluorescent intensity. In each graph, the bottom left quadrant represents the percentage of cells not showing AQPsexpression, while the bottom right quadrant represents the percentage of the cells expressing AQPs. A: negative control. B: AQP1 expression in CP cells (95.39\%). C: AQP4 expression in CP cells (92.21\%).

\section{Discussion}

The choroid plexuses are responsible for secreting the majority of CSF by a process leading to diffusion of water into the ventricles of the brain through AQP water channels $(17,18)$. Thus, the localization of AQPs in the CP is a useful development in the study of CSF secretion.
There is evidence that AQP1 and AQP4 are expressed in CPs (19). AQP1, which is highly expressed in the CP, has an undeniable role in mediating water transport across the apical membrane of the choroid plexus during CSF secretion. It has been demonstrated that $\mathrm{CP}$ exhibits the highest expression level of AQP1 compared to any other human tissue (20). Thus, both quantitative and qualitative studies of AQP1 expression in the CP are important. Studies have indicated that AQP1 is expressed in the apical membrane of the rat choroid plexus epithelium (16, 21). In the first part of this study, we examined the localization of AQP1 in CP cells by immunocytochemistry. The results demonstrated that $A Q P 1$ is only expressed in the cytoplasmic membrane of CP cells. Also, western blot analysis of AQP1 detected two bands of approximately 27 and $32 \mathrm{kDa}$ for this protein. Immunocytochemical and immunoblot data are in line with previous studies on AQP1 expression(5, 21, 22). In addition, flowcytometry was used to quantitatively examine AQP1 expression, and it was revealed that CP cells exhibit a high level of AQP1 (95.39\%). AQP4 is another water channel that is widely expressed in the brain (23). This protein is expressed in the basolateral membrane of many epithelia. In this study, we tested the hypothesis that AQP4 is expressed in the basolateral membrane of rat choroid plexus. In contrast to previous reports (11-14), using immunocytochemistry, we found that AQP4 is densely packed in the cytoplasm of CP cells with no expression in the membrane. This observation may suggest that these proteins may have no role in water transport across the basolateral membrane of $\mathrm{CP}$ cells. In contrast to previous reports $(12,24,25)$, western analysis revealed a single peptide of approximately 29 $\mathrm{kDa}$ for AQP4. In addition, quantitative examination of AQP4 expression by flowcytometry revealed that $92.21 \%$ of CP cells exhibit AQP4 expression. Thus, it is suggested that this protein may have an important role in CSF production.

AQP5 is also a member of the aquaporin subfamily. AQP5 mRNA has been detected in the rat brain using RT- 
Nazari Z et al.

PCR (29). However, the distribution of AQP5 in the CP has not been previously reported. In the present study, AQP5 expression, as another AQP water channel, was examined in the CP cells. Immunocytochemical analysis was applied to examine the localization of AQP5 in rat lateral ventricles CP. These results showed that AQP5 is weakly expressed in the cytoplasm of CP cells (Figure $2 \mathrm{C}$ ). Western analysis using AQP5 antibody recognized a pale band of approximately $28 \mathrm{kDa}$ (Figure 3). The low level of AQP5 expression in the cytoplasm is probably an indication of its lack of involvement in water transport as a whole, suggesting the involvement of other AQP channels in the CP. In conclusion, by immunocytochemistry immunoblotting and quantitative flowcytometry, AQP4 was abundantly detected in the rat lateral ventricles CP. These observations may suggest the prominent role of AQP4 in CSF secretion. However, as no expression of AQP4 or AQP5 was detected in cell membranes, thus, the route by which water crosses the basolateral membrane of CP cells remains to be determined.

\section{Acknowledgements}

This project was performed in the Laboratory of Cell and Developmental Biology at Kharazmi University and the authors wish to thank Prof. Shahrbanoo Oryan for her support.

\section{Authors' Contributions}

All authors contributed equally to this study.

\section{Financial Disclosure}

All authors had the same financial interest regarding this manuscript.

\section{Funding/Support}

This project was part of the MSc thesis of Ms Zahra Nazari. For this reason, the authors wish to thank the Faculty of Biological Science of Kharazmi University as well as the financial support of the Razi Herbal Medicine Research Center at the Lorestan University of Medical Sciences.

\section{References}

1. King LS, Agre P. Pathophysiology of the aquaporin water channels. Annu Rev Physiol.1996;58:619-48.

2. Lehmann GL, Gradilone SA, Marinelli RA. Aquaporin water channels in central nervous system. Curr Neurovasc Res. 2004;1(4):293303.

3. Venero JL, Vizuete ML, Machado A, Cano J. Aquaporins in the central nervous system. Prog Neurobiol. 2001;63(3):321-36.

4. Speake T, Whitwell C, Kajita H, Majid A, Brown PD. Mechanisms of CSF secretion by the choroid plexus. Microsc Res Tech. 2001;52(1):49-59.

5. Speake T, Freeman LJ, Brown PD. Expression of aquaporin 1 and aquaporin 4 water channels in rat choroid plexus. Biochim Bio- phys Acta. 2003;1609(1):80-6.

6. McCoy E, Sontheimer H. MAPK induces AQP1 expression in astrocytes following injury. Glia. 2010;58(2):209-17.

7. Zhang D, Vetrivel L, Verkman AS. Aquaporin deletion in mice reduces intraocular pressure and aqueous fluid production. J Gen Physiol. 2002;119(6):561-9.

8. Nielsen S, Frokiaer J, Marples D, Kwon TH, Agre P, Knepper MA. Aquaporins in the kidney: from molecules to medicine. Physiol Rev. 2002;82(1):205-44.

9. Frigeri A, Gropper MA, Umenishi F, Kawashima M, Brown D, Verkman AS. Localization of MIWC and GLIP water channel homologs in neuromuscular, epithelial and glandular tissues. J Cell Sci. 1995;108 ( Pt 9):2993-3002.

10. Venero JL, Vizuete ML, Ilundain AA, Machado A, Echevarria M, Cano J. Detailed localization of aquaporin-4 messenger RNA in the CNS: preferential expression in periventricular organs. Neuroscience.1999;94(1):239-50.

11. Hasegawa H, Ma T, Skach W, Matthay MA, Verkman AS. Molecular cloning of a mercurial-insensitive water channel expressed in selected water-transporting tissues. J Biol Chem. 1994;269(8):5497500.

12. Nielsen S, Nagelhus EA, Amiry-Moghaddam M, Bourque C, Agre P, Ottersen OP. Specialized membrane domains for water transport in glial cells: high-resolution immunogold cytochemistry of aquaporin-4 in rat brain. J Neurosci. 1997;17(1):171-80.

13. Yang M, Gao F, Liu H, Yu WH, He GQ, Zhuo F, et al. Immunolocalization of aquaporins in rat brain. Anat Histol Embryol. 2011;40(4):299-306.

14. Skjolding AD, Rowland IJ, Sogaard LV, Praetorius J, Penkowa M, Juhler M. Hydrocephalus induces dynamic spatiotemporal regulation of aquaporin-4 expression in the rat brain. Cerebrospinal Fluid Res. 2010;7:20.

15. Zheng W, Zhao Q, Graziano JH. Primary culture of choroidal epithelial cells: characterization of an in vitro model of blood-CSF barrier. In Vitro Cell Dev Biol Anim. 1998;34(1):40-5.

16. Nielsen S, Smith BL, Christensen EI, Agre P. Distribution of the aquaporin CHIP in secretory and resorptive epithelia and capillary endothelia. Proc Natl Acad Sci U S A.1993;90(15):7275-9.

17. Johanson CE, In PM, Conn Ed. Ventricles and cerebrospinal fluid. Neurosci in Med.1995:171-96.

18. Johanson CE, Duncan JA, 3rd, Klinge PM, Brinker T, Stopa EG, Silverberg GD. Multiplicity of cerebrospinal fluid functions: New challenges in health and disease. Cerebrospinal Fluid Res. 2008;5:10.

19. Zheng W, Chodobski A. The Blood-Cerebrospinal Fluid Barrier: Taylor and Francis group; 2005.

20. Mobasheri A, Marples D. Expression of the AQP-1 water channel in normal human tissues: a semiquantitative study using tissue microarray technology. Am J Physiol Cell Physiol. 2004;286(3):C52937.

21. Speake T, Kajita H, Smith CP, Brown PD. Inward-rectifying anion channels are expressed in the epithelial cells of choroid plexus isolated from ClC-2 'knock-out' mice. J Physiol. 2002;539(Pt 2):385-90.

22. Badaut J, Lasbennes F, Magistretti PJ, Regli L. Aquaporins in brain: distribution, physiology, and pathophysiology.JCereb Blood Flow Metab. 2002;22(4):367-78.

23. Rash JE, Yasumura T, Hudson CS, Agre P, Nielsen S. Direct immunogold labeling of aquaporin-4 in square arrays of astrocyte and ependymocyte plasma membranes in rat brain and spinal cord. Proc Natl Acad Sci U S A.1998;95(20):11981-6.

24. Terris J, Ecelbarger CA, Marples D, Knepper MA, Nielsen S. Distribution of aquaporin-4 water channel expression within rat kidney. Am J Physiol. 1995;269(6 Pt 2):F775-85.

25. Yamamoto N, Yoneda K, Asai K, Sobue K, Tada T, Fujita Y, et al. Alterations in the expression of the AQP family in cultured rat astrocytes during hypoxia and reoxygenation. Brain Res Mol Brain Res. 2001;90(1):26-38. 